\title{
Questes
}

vestes Revue pluridisciplinaire d'études médiévales

43 | 2021

Domestiquer la nature

\section{« Dorenlot » : chant des bergers, voix de la nature}

\section{Charlotte Guiot}

\section{(2) OpenEdition}

\section{Journals}

Édition électronique

URL : https://journals.openedition.org/questes/5828

DOI : $10.4000 /$ questes.5828

ISSN : 2109-9472

\section{Éditeur}

Les Amis de Questes

\section{Édition imprimée}

Date de publication : 30 juillet 2021

Pagination : 114-129

ISSN : 2102-7188

\section{Référence électronique}

Charlotte Guiot, « "Dorenlot » : chant des bergers, voix de la nature », Questes [En ligne], 43 | 2021, mis en ligne le 30 octobre 2021, consulté le 10 novembre 2021. URL : http://journals.openedition.org/ questes/5828; DOI : https://doi.org/10.4000/questes.5828 


\title{
« Dorenlot» : chant des bergers, voix de la nature
}

\section{Charlotte GUIOT}

\author{
Université Grenoble-Alpes
}

« Le mestier de la garde des oeilles est moult honorable et de grant auctorité. Ce peult-on prouver appertement par nature et par la saincte escripture $^{1} \gg$. C'est ainsi que Jean de Brie ouvre la présentation du métier de berger dans son traité du Bon berger, probablement écrit pour le roi Charles V au XIV ${ }^{\text {e }}$ siècle. «Honorable», les auteurs des pastourelles, poèmes courts dont les héroïnes sont souvent présentées comme des filles faciles à séduire, semblent en douter. Si l'autorité du berger, incarnant alors le bon dirigeant, est bien l'objet de certains textes religieux ou encore de pastorales politiques, ce personnage illustre aussi la légèreté et l'insouciance, comme c'est le cas dans le Jeu de Robin et Marion, où les héros se livrent à de nombreux jeux, danses et autres réjouissances. Figure variée aux caractéristiques changeantes, le berger connaît au Moyen Âge un succès qui ne se démentira pas par la suite.

Ce statut protéiforme et cette capacité à traverser les genres et les âges, le berger les doit peut-être à sa position particulière d'intermédiaire. $\mathrm{Si}$, par son statut social, il incarne une forme d'humilité, il est également le maître du troupeau. Cette position spécifique explique peut-être le

\footnotetext{
${ }^{1}$ Jean de Brie, Le bon berger, ou Le vray régime et gouvernement des bergers et bergères composé par le rustique Jehan de Brie, le bon berger (1541), éd. Paul Lacroix, Paris, Isidore Liseux, 1879, p. 38.
} 
succès qu'il rencontrera à partir du $\mathrm{XIV}^{\mathrm{e}}$ siècle, sous Charles VI, dans les représentations du pouvoir ${ }^{2}$. En raison du lieu où il évolue, il se tient à mi-chemin entre monde sauvage et monde civilisé ; il est le personnage du saltus, lieu que Fabrice Mouthon définit comme 1' « espace de compromis entre l'homme et les forces de la nature ${ }^{3} »$. Guidant les troupeaux, il vit avec et dans la nature, espace qui lui est familier et qu'il maitrise partiellement. La littérature pastorale, par la représentation idyllique qui est proposée de la nature, presque exclusivement printanière et rieuse, offre le portrait d'un personnage évoluant en harmonie dans un environnement protecteur et familier. Paradoxalement, le saltus est donc pour les bergers un lieu connu et maitrisé, voire domestiqué en partie.

Le chant, pratique caractéristique de ces personnages, s'inscrit au sein d'une harmonie naturelle, se fait écho des voix de cette nature et traduit l'attitude ambivalente de la cour à l'égard de cette dernière, lieu fantasmé, recomposé et regretté, espace obscur à déchiffrer, mystère à préserver. La présente étude se concentrera principalement sur des considérations poétiques, afin de mieux comprendre comment le texte construit un cadre harmonieux. Si ce dernier peut bien évidemment avoir des fins politiques, elles ne seront pas l'objet principal de notre propos. Nous chercherons ainsi à déterminer comment la littérature pastorale fait

\footnotetext{
${ }^{2}$ Colette Beaune, «Opération bergère », dans Jeanne d'Arc, Paris, Perrin, 2004, p. 115-133. Sur les liens entre pouvoir et pastorat, voir aussi Jacques Dalarun, «Pastorat», dans Gouverner c'est servir, Paris, Alma, 2012, p. 292-306 et Michel Foucault, Sécurité, territoire, population. Cours au Collège de France. 1977-1978, Paris, Gallimard/EHESS/Éditions du Seuil, 2004. Sur l'opposition entre berger-bon souverain et loup-tyran, voir Denis Hüe, «Le berger à la fin du Moyen Âge. Remarques sur une figure trifonctionnelle», dans Remembrances et resveries. Hommage à Jean Batany, dir. Huguette Legros, Denis Hüe, Joël Grisward et Didier Lechat, Orléans, Paradigme, 2006, p. 117-138, ici p. 129.

${ }^{3}$ Fabrice Mouthon, Le sourire de Prométhée. L'homme et la nature au Moyen Âge, Paris, La Découverte, 2017, p. 19.
} 
de ce chant des bergers, à la fois familier et étranger, un élément clé d'une relation idyllique rêvée.

Nous étudierons ici plus particulièrement les pastourelles ${ }^{4}$, écrites pour la plupart au début du XIII ${ }^{\mathrm{e}}$ siècle, le Jeu de Robin et Marion d'Adam de la Halle $^{5}$, pièce de théâtre du milieu du XIII ${ }^{\mathrm{e}}$ siècle développant le schéma de la pastourelle et accompagnée de partitions, et Regnault et Janneton de René d'Anjou ${ }^{6}$, forme narrative en vers du $\mathrm{XV}^{\mathrm{e}}$ siècle, écrite à l'occasion du mariage du roi avec sa nouvelle épouse, Jeanne de Laval. À travers ce corpus divers et ancré dans des époques différentes, nous pourrons ainsi explorer les réalisations multiples de ce lien de l'homme à la Nature en cherchant à savoir s'il est constant et caractéristique de la pastorale en général.

Le rapprochement de ces différents textes ne va en effet pas de soi. Les bergères des pastourelles sont des personnages comiques, dont on moque la naïveté et l'appétit sexuel et que l'on renvoie à une classe sociale distincte et inférieure. À l'inverse de ces figures types, Regnault et Janneton incarnent les doubles pastoraux, mais courtois, du roi René d'Anjou et de son épouse Jeanne de Laval. Cette évolution témoigne peut-être d'une forme de valorisation d'un retour à un mode de vie humble à la fin du Moyen Âge, célébré notamment par le Dit de Franc Gontier de Philippe de Vitry au XIV siècle.

La musique nous permettra de plus de dégager une cohérence entre ces différents textes : les bergères des pastourelles sont caractérisées par

\footnotetext{
${ }^{4}$ Karl Bartsch, Romances et pastourelles françaises des XII ${ }^{e}$ et XIII siècles, Genève, Slatkine Reprints, 1973 ; Jean-Claude Rivière, Pastourelles, volumes 1-3, Genève, Droz, 1974-1976.

5 Adam de la Halle, Le Jeu de Robin et Marion, dans Euvres complètes, éd. Pierre-Yves Badel, Paris, Librairie Générale Française, coll. « Lettres gothiques », 1995.

${ }^{6}$ René d'Anjou, Regnault et Janneton, éd. Gilles Roussineau, Genève, Droz, coll. « Textes littéraires français », 2012.
} 
leur chant et les bergers se réunissent lors de fêtes musicales et de danses. Adam de la Halle prolonge cette représentation en faisant de son jeu une œuvre musicale à part entière. Enfin, si les bergers de Regnault et Janneton ne chantent pas et ne jouent pas de musique, ils portent un intérêt prononcé aux oiseaux ${ }^{7}$, dont l'apparition est soigneusement insérée au sein du concert de la Nature.

\section{Le berger, figure intermédiaire entre l'homme et la Nature}

\section{Le berger, figure humanisée de la Nature ?}

Le berger des pastourelles vit et se fond au sein de la Nature, au point que le poète convoque une même palette pour dépeindre les bergères et le cadre naturel où elles évoluent. La couleur de la rose qui revient régulièrement pour qualifier le teint des bergères dresse ainsi un parallèle entre le personnage et la végétation. «La colour/ ot freche com rose en $\mathrm{mai}^{8}$ » déclare ainsi le narrateur d'une pastourelle au sujet de la bergère rencontrée. Un autre poète utilise le verbe « rouzoier $^{9} »$, que le dictionnaire Godefroy définit comme «avoir la couleur de la rose, être rouge, se couvrir d'une teinte rouge», mais aussi "être mouillé de rosée ${ }^{10} »$. Au-delà de la couleur, les lèvres de la bergère, se couvrant de rosée dans une description érotisée, sont soumises au même phénomène naturel que les plantes. Le corps de la bergère se fait ainsi floral,

\footnotetext{
${ }^{7}$ Au sujet de l'intérêt de René d'Anjou pour les oiseaux, voir Sophie O. Poitral, « La nomination de l'oiseau chez René d'Anjou », dans Déduits d'oiseaux au Moyen Âge, dir. Chantal Connochie-Bourgne, Aix-en-Provence, PUP, 2009, p. 259-268. URL : https://books.openedition.org/pup/4294 (consulté le 6 septembre 2018).

${ }^{8}$ Karl Bartsch, Romances et pastourelles françaises des XII et XIII siècles, partie 2, pastourelle 5, p. 108, v. 5-6.

${ }^{9}$ Ibid., partie 2, pastourelle 45, p. 165, v. 14.

10 «Rosoier », dans Frédéric Godefroy, Dictionnaire de l'ancienne langue française et de tous ses dialectes $d u I X^{e}$ au $X V^{e}$ siècle, Paris, Librairie des Sciences et des Arts, 1938, tome 7, p. 242.
} 
brouillant la limite entre l'humain et la plante. La réalisation des nombreuses couronnes de fleurs dont s'ornent bergers et bergères ${ }^{11}$ renforce cette végétalisation du corps.

\section{Le berger et le poète, écouter la Nature}

Cette proximité, pour ne pas dire fusion, permet au berger d'entretenir un lien étroit avec la Nature, lien qui pourrait également être celui du poète. S'ils chantent tous deux, ils sont également des figures de l'écoute, prêtant une oreille attentive à la Nature et à ses secrets. Le poète de Regnault et Janneton refuse de trop parler pour ne pas être infidèle à la réalité du chant des oiseaux, déclarant « Sur quoy ne sçay plus autre rien qu'en die, /si sollassable est leur chant d'escouter /et leur maintien, sans ce que $\mathrm{j}^{\prime}$ en mesdie ${ }^{12}$ ». Le blanc séparant dans le manuscrit les deux strophes fait alors éclater ce silence du poète. Le berger qui apparaît ensuite adopte une posture similaire : «doulcement escouter/va pas a pas et les verbes noter /des oisillons pour au vray raporter ${ }^{13} »$. Il s'établit ainsi un rapport esthétique au monde, semblable à celui de Marion qui, lors de sa rencontre avec le chevalier, se soucie du chant des oiseaux là où son

\footnotetext{
${ }^{11} \mathrm{Si}$ les bergers comme les bergères peuvent recevoir des couronnes de fleurs, ce sont ces dernières qui sont érotisées par le regard du narrateur décrivant la scène. Voir Geri L. Smith, The Medieval French Pastourelle Tradition: Poetic Motivations and Generic Transformations, Gainesville, University Press of Florida, 2009, p. 45 et sq. Les bergers ne sont pas les seuls à s'offrir des couronnes de fleurs, c'est une pratique courante au Moyen Âge, qui est décrite par exemple dans le Mesnagier de Paris, éd. Georgina E. Brereton et Janet M. Ferrier, trad. Karin Ueltschi, Paris, Librairie Générale Française, coll. «Lettres gothiques », Paris, 1994, p. 24 : « Mais saichiez que je n'en pren pas desplaisir, mais plaisir, en ce que vous avrez a labourer rosiers, a garder violectes, faire chappeaulx, et aussi en vostre dancer et en vostre chanter ».

${ }^{12}$ René d'Anjou, Regnault et Janneton, éd. cit., p. 48, v. 48-50.

${ }^{13}$ Ibid., p. 50, v. 92-94. Cette attitude du berger, soulignant les liens entre la musique et le chant des oiseaux, pourrait nous évoquer le travail de compositeurs à venir, Clément Janequin au siècle suivant, mais aussi, au sein d'une histoire de la musique au long cours et bien plus proche de nous, Olivier Messiaen (on songe par exemple au Réveil des oiseaux de 1953). Je remercie chaleureusement le professeur Denis Hüe de m'avoir signalé ce rapprochement ainsi que la référence au Mesnagier de Paris.
} 
interlocuteur cherche uniquement des proies: «Encore $i$ a en ces buissons /Cardonneruel[e]s et pinçons /Qui mout cant joliement ${ }^{14} »$.

Cette attention particulière des bergers et poètes à leur environnement explique peut-être qu'ils soient souvent représentés comme dépositaires d'un savoir particulier, dont ils se font les intermédiaires auprès de la cour. Que ce soit dans le traité du Bon berger mentionné en introduction ou dans le Kalendrier des bergers, le personnage pastoral est en effet celui qui sait déchiffrer les signes ${ }^{15}$.

\section{Le rêve d'une relation harmonieuse : l'ouverture de Regnault et Janneton}

Caractérisés par leur jeunesse, sachant écouter la Nature, les bergers ne sont pas sans rappeler le mythe de l'âge d'or dont Jean de Meung propose une description dans le Roman de la Rose ${ }^{16}$. Outre le cadre de vie, certains détails rappellent les mœurs des héros pastoraux. Les habitants de l'âge d'or ne seraient-ils pas des bergers puisqu'ils s'habillent de « robes de lainnes", font avec les fleurs de « biaus chapelez» et dansent la " $\operatorname{karole}^{17}$ » ? Il est possible en tout cas de rapprocher ces deux types de figures, incarnant, en partie du moins, le rêve d'une vie heureuse au sein de la Nature.

Cette relation idéale, rêvée, se traduit lors de la rencontre du chant des bergers avec les voix de la Nature, le berger participant ainsi à

\footnotetext{
${ }^{14}$ Adam de la Halle, Le Jeu de Robin et Marion, éd. cit., p. 208, v. 27-29.

${ }^{15}$ Voir à ce sujet Denis Hüe, «Le berger à la fin du Moyen Âge. Remarques sur une figure trifonctionnelle», art. cit., p. 135 : «le berger maîtrise ainsi le temps météorologique et le comput, l'heure aussi bien que l'année. Il maitrise aussi l'espace, capable qu'il est, en pleine nuit, de reconnaître les points cardinaux. Cette compréhension du temps et de l'espace s'accompagne d'une compréhension profonde du microcosme $»$.

${ }^{16}$ Guillaume de Lorris et Jean de Meung, Le Roman de la rose, éd. Armand Strubel, Paris, Librairie Générale Française, coll. « Lettres gothiques », 2012, p. 456-460.

${ }^{17}$ Ibid., p. 458 , v. 8391 ; p. 460 , v. 8424 , v. 8443.
} 
l'harmonie du monde. Le prologue de Regnault et Janneton introduit les figures humaines au sein d'un véritable concert de la Nature où cette dernière apparaît sous les traits d'un orgue naturel, plus harmonieux que tout instrument humain. Il présente dans un premier temps l'accord de la Nature, puis y intègre délicatement des figures humaines. Évoquant le chant des oiseaux, le poète déclare ainsi :

Car c'est, ce croy, trop plus doulce armonie /que d'instrumens, desquelz le son eslie /les cuers des gens, car leur voix tresserie /est plus a droit et par fine maistrie/bien reiglee que nul ton d'organie / par musique tel qu'on saiche noter ${ }^{18}$.

La comparaison est établie entre harmonie naturelle et harmonie humaine, la première l'emportant largement sur la seconde. D'obscure et insaisissable par sa supériorité, la Nature devient lisible au prisme d'une connaissance humaine, celle du poète et du musicien.

Les comparaisons entre musique naturelle et artificielle ne se limitent pas à la mention de l'orgue. Tels un chœur polyphonique, les oisillons « chantent a contre et a teneur ${ }^{19}$ », et tels des trouvères ou des troubadours, «Amours ja les occist ${ }^{20}$ ». La grenouille ${ }^{21}$, à l'image d'un chef d'orchestre ou d'un tambour, vient donner le rythme de la mélodie qui s'élève avec la naissance du printemps.

D'autre part, dedans le bouillon /chante le petit grenoillon, /ouquel partout en a foisson, /en l'eau coye, pres l'oresson, /qui donne une

\footnotetext{
${ }^{18}$ René d'Anjou, Regnault et Janneton, éd. cit., p. 48, v. 51-56.

${ }^{19}$ Ibid., p. 46, v. 28.

${ }^{20}$ Ibid., p. 46, v. 24.

${ }^{21}$ Sur le jeu littéraire introduit par les animaux mentionnés, voir Michelle Szkilnik, «Le jeu littéraire dans Regnault et Janneton», dans Sens, Rhétorique et Musique. Études réunies en hommage à Jacqueline Cerquiglini-Toulet, dir. Sophie Albert, Mireille Demaules, Estelle Doudet, Sylvie Lefèvre, Christopher Lucken et Agathe Sultan, Paris, Honoré Champion, 2015, p. 207-208.
} 
proporciion /de voix doulce haussant son ton /lent tout apert par envoisie. ${ }^{22}$

Le rejet de l'adjectif «lent» crée une hésitation sur le sens de « haussant le ton » qui pourrait signifier une augmentation du volume ou un changement de hauteur, le passage vers une note plus aigüe. Cette hésitation crée un suspens, une attente, qui est soudain suivie d'une précipitation avec l'apparition au vers suivant de l'adjectif. L'enjambement vient mimer l'accélération du rythme opérée par la grenouille, rendue ainsi proche du public par le travail métrique.

L'ouverture de Regnault et Janneton se construit sur un crescendo progressif mimant le retour du printemps et aboutissant à l'apparition de figures humaines, à la fois distinctes et pourtant pleinement intégrées au cadre champêtre. Après une première strophe sans mention d'élément sonore, la mélodie est d'abord portée par «les oisillons ${ }^{23}$ », illustrant ainsi un chant naissant, émergeant avec les beaux jours. Ce chant s'étend ensuite géographiquement avec le départ des oisillons qui quittent le nid et vont « les buissons hanter ${ }^{24} »$. À cette expansion horizontale répond, à la strophe suivante, un mouvement vertical porté par l'envol de nouveaux oiseaux, «calandes » et « alloetes », les unes commençant à « volleter », les autres « en l'air hault monter ${ }^{25} »$. Les voix se multiplient grâce à l'arrivée d'autres oiseaux : « le merle mauviz, le pinson ${ }^{26}$ » apparaissent à la strophe IX, puis se diversifient en convoquant à la strophe $\mathrm{X}$ une série d'insectes et finalement la grenouille (strophe XI) et le pigeon, dernier représentant de cette faune chantante et animal type de la symbolique amoureuse, annonçant les débats amoureux qui se développeront dans la

\footnotetext{
${ }^{22}$ René d'Anjou, Regnault et Janneton, éd. cit., p. 50, v. 71-77.

${ }^{23}$ Ibid., p. 46, v. 12.

${ }^{24}$ Ibid., p. 48 , v. 30.

${ }^{25}$ Ibid., p. 48, v. 36-38.

${ }^{26}$ Ibid., p. 48, v. 57.
} 
suite du texte. Par cette succession de petits tableaux, cette accumulation progressive de voix, le poète compose une véritable polyphonie, donne à voir et à entendre cet orgue de la Nature, instrument puissant au souffle continu, aux matériaux et sonorités si divers ${ }^{27}$. Or, l'orgue est par excellence l'instrument religieux, élément à part entière des églises et des cathédrales, permettant d'exprimer la louange des hommes. Grâce à cette rencontre harmonieuse entre les voix hétérogènes de la Nature, le poète célèbre l'ordre naturel et divin du monde ${ }^{28}$.

L'ouverture de Regnault et Janneton propose donc une peinture idéale de la Nature, revisitant en le déployant le motif habituel de la reverdie et recréant une polyphonie naturelle pour mettre en avant l'harmonie de l'univers décrit. Par les schémas métriques et strophiques, le poète renforce cette impression en employant une structure subtilement close sur elle-même, s'étalant sur quatre strophes suivant le modèle : aaaaaab aaaaaab bbbbbba bbbbbba.

L'arrivée de l'homme se traduit par un bref changement de structure avec seulement deux strophes répétant le modèle aaaaaab, auquel succède immédiatement un retour à la structure initiale, avant que ne débutent de nouveaux changements. L'homme est ainsi inscrit dans ce cycle naturel. Cette intégration est redoublée dans la matérialité du manuscrit par l'inscription du texte au sein d'enluminures représentant la

\footnotetext{
${ }^{27}$ Sur l'orgue, voir Denis Hüe, «La machine orgue», dans Engins et machines : l'imaginaire mécanique dans les textes médiévaux, dir. Fabienne Pomel et Anne Berthelot, Rennes, PUR, 2015, p. 87-103, notamment p. 98 : "Construit de bois, de métal et de cuir, nécessitant l'eau et le feu pour sa construction, ancré dans la maçonnerie et traversé par l'air qui l'anime, l'orgue est à lui seul une cosmogonie, rassemblant le meilleur de la nature pour que l'homme, l'actionnant, fasse son action de grâce et exalte l'humilité du Christ incarné »".

${ }^{28}$ Michel Zink, Nature et poésie au Moyen Âge, Paris, Fayard, 2006, p. 192 : «Le mystère de l'harmonie musicale, produite par des sons et des instruments pourtant différents, frappe beaucoup le Moyen Âge ; il lui sert en particulier à décrire la louange qui monte vers Dieu de la nature entière et de toutes ses créatures dans leur diversité ».
} 
nature. Bien que la voix du poète se détache sur le décor naturel de la page, elle y est parfaitement intégrée, s'inscrit au cœur de la Nature ${ }^{29}$. Alors que les bergères des pastourelles semblaient se confondre avec les fleurs, les personnages de cette ouverture constituent des entités distinctes mais s'accordent avec la Nature pour participer à une harmonie universelle, pour ajouter leur contribution à une polyphonie mélodieuse permettant la célébration unanime du nouveau couple royal, Jeanne et René, qui apparaîtra dans les vers suivants. La représentation idyllique proposée ici permet de porter une promesse politique de paix et d'entente, dépassant les sphères humaines pour permettre une réconciliation avec le cosmos entier.

\section{La langue du berger}

\section{L'onomatopée : une langue naturelle?}

Cette intégration musicale à la Nature n'est-elle pas caractérisée dans les pastourelles et chez Adam de la Halle par l'usage de l'onomatopée, langue inarticulée qui pourrait faire signe vers une parole première et non-civilisée ? On relève en effet que les onomatopées apparaissent dans 34 des 182 pastourelles éditées par Bartsch $^{30}$, soit environ dans une pastourelle sur cinq. Elles en constituent ainsi un élément caractéristique. Loin des subtilités de la langue courtoise, la langue $\mathrm{du}$ berger serait une forme de babillage enfantin, production spontanée, fruit des sentiments. Dans le tableau reproduit par Christopher

\footnotetext{
${ }^{29}$ René d'Anjou, Regnault et Janneton, éd. cit., annexes, planches I et II, reproduction des folios du manuscrit Saint-Pétersbourg, Bibliothèque nationale de Russie, Fr. Q. p. XIV. 1, fol. $1 \mathrm{r}$ et fol. $3 \mathrm{r}$.

${ }^{30}$ Karl Bartsch, Romances et pastourelles françaises des XII et XIII ${ }^{e}$ siècles, éd. cit.
} 
Lucken ${ }^{31}$ d'après les Questions sur l'interjection, on ne relève que l'interjection « eya » qui ponctue l'une des pastourelles mais elle semble exprimer l'espoir du chevalier et non de la bergère ${ }^{32}$. En revanche, les autres onomatopées, et donc celles prononcées par les bergers, ne figurent pas dans le tableau. On peut supposer que c'est parce qu'elles ne renvoient pas à des cris mais au chant, dont Christopher Lucken souligne par ailleurs l'importance dans la littérature médiévale ${ }^{33}$.

Le chant des bergers pourrait ainsi, dans l'imaginaire de la cour, mais aussi dans notre imaginaire contemporain, s'apparenter à une voix des origines. L'usage de l'onomatopée pour retranscrire ce chant met en avant sa simplicité et son authenticité.

La figure qui permettrait le mieux de signifier la mélopée est l'onomatopée : elle relève de la mélodie pure par son sémantisme vide, ou plus exactement l'onomatopée, sous la forme de l'interjection, signifie par affect, non par concept, pour reprendre une distinction présente chez les grammairiens du XIII ${ }^{\mathrm{e}}$ siècle. ${ }^{34}$

écrit Jean-Marie Fritz dans un article consacré au chant de l'oiseau.

Caractérisés par leur chant simple, signifiant et exprimant leurs sentiments, nos bergers sont peut-être des figures proches des premiers hommes décrits par Rousseau dans son Essai sur l'origine des langues.

\footnotetext{
${ }^{31}$ Christopher Lucken, «Éclats de la voix, langage des affects et séductions du chant. Cris et interjections à travers la philosophie, la grammaire et la littérature médiévales » dans Haro! Noël ! Oyé ! Pratiques du cri au Moyen Âge, dir. Didier Lett et Nicolas Offenstadt, Paris, Publication de la Sorbonne, 2003, p. 195.

${ }^{32}$ Karl Bartsch, Romances et pastourelles françaises des XII et XIII ${ }^{e}$ siècles, éd. cit., partie 2, pastourelle 12, p. 118-120.

${ }^{33}$ Christopher Lucken, «Éclats de la voix, langage des affects et séductions du chant », art. cit., p. 196.

${ }^{34}$ Jean-Marie Fritz, «Le chant de l'oiseau est-il une musique ? " dans D'ailes et d'oiseaux au Moyen Âge: langue, littérature et histoire des sciences, dir. Claude Thomasset, Paris, Honoré Champion, 2016, p. 235.
} 
Les onomatopées renvoient ainsi à une simple mélodie, expression d'un sentiment.

Le terme «Dorenlot» qui revient fréquemment dans les pastourelles est à ce titre intéressant. Il pourrait avoir avec le verbe «dorloter», que nous utilisons encore aujourd'hui, une origine commune. Le dictionnaire Godefroy propose en effet comme entrée «dorenlot - dorelot », renvoyant au chant, mais aussi «doreloter », ancêtre de notre « dorloter ». Le TLFi rapproche de plus l'étymologie de «dorloter» de celle de «doreloterie » au sujet de laquelle il indique « dérivé de l'ancien français dorelot, dorenlot, "exclamation servant de refrain dans les pastourelles" 35 ». L'onomatopée ne serait-elle donc pas un chant affectueux, une cajolerie mélodique ? Par ailleurs, son emploi est clairement placé du côté du chant et de la mélodie puisque l'on trouve dans une pastourelle l'expression "chantant un dorenlot ${ }^{36}$ ». Un glissement métonymique s'opère de la réalisation sonore du chant à sa désignation. On peut également y voir l'imitation du son des instruments. Ainsi lit-on chez Jean Bodel «Lors si chalemele, /Dorenlot aé ! ${ }^{37} »$. Abolissant la langue articulée, le chant des bergers se ferait ainsi incrustation d'un sentiment mélodieux, audible, poésie pure et originelle. Il serait une porte ouverte vers la Nature sauvage et le rêve d'une langue première.

Toutefois, ce chant marque également la distance qui sépare les bergers de la cour, et plus encore du lecteur contemporain. Il est en effet marqué par une obscurité autorisant peut-être une complicité grivoise de

\footnotetext{
35 « Dorloter» et « doreloterie », dans Trésor de la Langue Française informatisé.

${ }^{36}$ Karl Bartsch, Romances et pastourelles françaises des XII et XIII siècles, éd. cit., partie 2, pastourelle 22, p. 136.

37 Jean Bodel, « Contre le douz tans novel», dans Annette Brasseur, «Les Pastourelles de Jehan Bodel», dans Arras au Moyen Âge: histoire et littérature, dir. Marie-Madeleine Castellani, Arras, Artois Presses Universitaires, 1994, p. 291, v. $10-11$.
} 
l'auteur avec le public. Ainsi, dans la pastourelle anonyme 22, les bergers évoquent le "pickenpot», qui semble ici un hapax puisque le Dictionnaire Godefroy cite cette même pastourelle pour exemple et propose comme définition un laconique et expressif : «s.m : ? ${ }^{38} »$. Cette expression pourrait être rattachée à l'érotique «jeu de pic en pance» mentionné par Bodel dans l'une de ses pastourelles ${ }^{39}$. En créant une relation particulière entre les bergers et la Nature, le poète ne souligne-t-il pas par opposition la séparation irréductible entre cette dernière et le monde courtois, séparation qui autorisera, dans le cas de certaines pastourelles, la violence du narrateur et la réalisation forcée de ses désirs?

Dans une pastourelle du même auteur, « Les un pin verdoiant ${ }^{40} »$, le narrateur est exclu de la communauté pastorale par le refrain qui met en avant la relation entre les bergers amants. Les verbes introducteurs de paroles du refrain sont à l'imparfait, inscrivant les échanges de Robin et Marion dans la durée, là où le dialogue de Marion et du narrateur est retranscrit au passé simple, sous le signe de l'événement mais aussi du momentané. Ni le discours du narrateur, ni la séparation des bergers, lorsque Robin s'éloigne dans les bois, ne parviennent à briser le retour de ce refrain. La mélodie, conservée dans le chansonnier de Noailles ${ }^{41}$, construit en miroir la voix des deux héros pastoraux par une reprise du même motif mélodique, créant une forme de gémellité sonore venant souligner l'harmonie amoureuse au sein du couple et, par extension,

\footnotetext{
38 «Pickenpot» dans Frédéric Godefroy, Dictionnaire de l'ancienne langue française et de tous ses dialectes du IXe au XV siècle, op. cit., t. 6, p. 143.

39 Jean Bodel, «L'autrier quant chevauchoie», dans Annette Brasseur, «Les Pastourelles de Jehan Bodel », art. cit., v. 74. Je remercie chaleureusement Maxime Kamin pour m'avoir signalé ce possible rapprochement lors de ma communication.

40 Jean Bodel, «Les un pin verdoiant », dans Annette Brasseur, "Les Pastourelles de Jehan Bodel », art. cit., p. 280-287.

${ }^{41}$ Chansonnier de Noailles, Paris, BnF, ms français 12615, fol. $85 \mathrm{v}$.
} 
l'exclusion du narrateur qui finira par faire usage de la violence pour briser le lien entre les bergers. Contrairement à la polyphonie qui s'élève dans le Dit de Regnault et Janneton, le chant des bergers vient ici souligner leur irréductible étrangeté, celle de la Nature, étrangeté qui interdit peut-être des échanges plus pacifiés avec le monde courtois. Les pastourelles présentent ainsi une note dissonante et n'ont pas pour objectif la célébration politique d'une harmonie idéale.

\section{La tradition pastorale, altérité familière}

Cette étrangeté de la voix du berger est toutefois le fruit d'une création littéraire. Si les pastourelles sont liées à une tradition populaire ${ }^{42}$, on peut supposer que leurs traits archaïsants et popularisants sont parfois intentionnellement repris par les trouvères pour accentuer leur altérité. Ainsi, l'usage des onomatopées, faisant signe vers une pure musique - un hier du langage -, est-il statistiquement plus important dans les pastourelles attribuées à un auteur que dans les pastourelles anonymes ${ }^{43}$. Chez Bartsch, l'usage de l'onomatopée est deux fois plus répandu dans les pastourelles attribuées à un auteur que dans les pastourelles anonymes ${ }^{44}$. Comme l'indique Michel Zink au sujet de la littérature médiévale, les pastourelles se construisent en « trompe-l'œil ${ }^{45}$ » en renvoyant par l'artifice poétique à un passé perdu. Elles se situent ainsi

42 Pastourelles. I, Introduction à l'étude formelle des pastourelles anonymes françaises des XII et XIII siècles, textes du chansonnier d'Oxford, avec notes, éd. Jean-Claude Rivière, vol. 1, Genève, Droz, coll. «Textes littéraires français ", 1974 , p. 68.

${ }^{43}$ Anonymat et fond traditionnel ne se superposent pas, bien sûr, et il est très probable que les pastourelles anonymes aient été écrites, elles aussi, par des auteurs de cour. Le doute peut néanmoins subsister, ce qui n'est pas le cas des pastourelles attribuées à des auteurs dont les productions les rattachent au monde de la cour.

${ }^{44}$ Karl Bartsch, Romances et pastourelles françaises des XII et XIII ${ }^{e}$ siècles, éd. cit.

${ }^{45}$ Michel Zink, Le Moyen Âge et ses chansons ou Un passé en trompe-l'œil, Paris, Éditions de Fallois, 1996, p. 30. 
dans un entredeux, entre altérité du passé et proximité d'une tradition toujours vivante.

L'écriture pastorale s'inscrit avant tout dans une tradition poétique où les voix se font échos. On note par exemple la réapparition d'un même refrain d'un texte à l'autre. Les vers sur lesquels s'ouvre le Jeu de Robin et Marion « Robins m'aime, Robins m'a /Robins m'a demandee, si m'ara ${ }^{46}$ " apparaissent également dans le recueil de Bartsch, dans la pastourelle anonyme $71^{47}$ et dans la pastourelle de Perrins d'Angeco, « Au tens nouvel que cil oisel ${ }^{48} »$. Il est ainsi possible de supposer que certains refrains circulaient d'un texte à l'autre, jouant sur la mémoire des auditeurs en créant des liens entre différentes œuvres et superposant ainsi plusieurs voix au sein d'une même performance. Les pastourelles que Jean-Claude Rivière nomme «avec refrains», par opposition aux pastourelles « à refrain » dotées d'un refrain fixe ${ }^{49}$, fonctionnaient peutêtre comme une série de clins d'œil intertextuels. Nous aurions affaire, ici aussi, à ce que Michel Zink, étudiant le texte de la « bele Aélis », nomme « un pot-pourri de refrains ${ }^{50} »$. La pastourelle repose de plus, comme 1'a montré Paul Zumthor, sur le retour de motifs et de formules, constituant un «noyau original, irréductible et complexe ${ }^{51} »$. Par ces jeux d'échos, la littérature pastorale est donc dotée d'un caractère circulaire, semblable à un ailleurs en perpétuel recommencement, réalisation partielle du rêve

\footnotetext{
${ }^{46}$ Adam de la Halle, Le Jeu de Robin et Marion, éd. cit., p. 206, v. 1-2.

${ }^{47}$ Karl Bartsch, Romances et pastourelles françaises des XII et XIII siècles, éd. cit., partie 2, pastourelle 71, p. 197, v. 50-53.

${ }_{48}$ Ibid., partie 3, pastourelle 42, p. 295, v. 26-27.

49 Pastourelles. I, Introduction à l'étude formelle des pastourelles anonymes françaises des XII et XIII siècles, textes du chansonnier d'Oxford, avec notes, éd. cit., p. 61 .

${ }^{50}$ Michel Zink, Le Moyen Âge et ses chansons ou Un passé en trompe-l'œil, op. cit., p. 166.

51 Paul Zumthor, "L'exemple des pastourelles ", dans Langues et techniques poétiques à l'époque romane (XI -XIII siècles), Paris, Klincksieck, 1963, p. 145-150.
} 
d'un passé idyllique éternellement jeune, à l'image de ces bergers enfants. Ils contribuent à créer un sentiment de familiarité entre l'auditeur et l'ailleurs qui lui est décrit, étranger et pourtant connu.

Alors que l'homme sauvage des romans de chevalerie semble l'émanation d'une nature hostile et constitue une figure d'altérité frappante pour le public courtois ${ }^{52}$, le berger, bien que rustique, présente une version plus accueillante et hospitalière de la Nature. Par son intermédiaire, le poète offre à la cour le rêve d'une vie en accord avec le monde, résonnant avec la nostalgie d'un âge d'or perdu et d'une proximité passée avec la Nature. Prenant place dans une tradition littéraire qui désigne le caractère utopique de ce rêve et renvoie le public aristocratique à ses contradictions entre désir de maitrise et aspiration aux origines, cette inscription dans les mémoires communes permet également de créer une proximité entre le public et ces personnages qui entrent à la cour grâce au jongleur ou au trouvère. Ces derniers incarnent leurs voix le temps d'une performance, et font toucher du doigt le rêve d'une harmonie entre hommes et Nature.

Le jeu avec cette mémoire commune, passant par la reprise de certains éléments, permet de tisser un lien des pastourelles du XIII ${ }^{\mathrm{e}}$ siècle à Regnault et Janneton au $\mathrm{XV}^{\mathrm{e}}$ siècle. Si les bergers sont toujours intégrés à la Nature et participent à une harmonie illustrée notamment par la musique, leur positionnement évolue. Dans la pastourelle, la bergère incarne son environnement, devenant alors pour le narrateur désirant un

\footnotetext{
${ }^{52}$ On peut penser par exemple au vilain rencontré par Calogrenant et Yvain : Chrétien de Troyes, Le Chevalier au lion, éd. David F. Hult, Paris, Librairie Générale Française, coll. «Lettres gothiques », 1994, v. 286-407 et v. 792-797. Voir aussi Micheline de Combarieu, "Image et représentation du vilain dans les chansons de geste (et dans quelques autres textes médiévaux)», dans Exclus et systèmes d'exclusion dans la littérature et la civilisation médiévales, Aix-en-Provence, PUP, 1978. URL : http://books.openedition.org/pup/3195 (consulté le 12 février 2019).
} 
double de la Nature avec lequel s'unir ${ }^{53}$, parfois par la force. Son chant est carmen, charme irrésistible. Dans Regnault et Janneton, la voix du poète célèbre l'ordre naturel et divin, présentant le roi et la reine d'Anjou au sein d'un univers pacifié et dont l'harmonie se traduit par la douce mélodie de la Nature. Le corpus pastoral médiéval, s'il possède une certaine cohérence, est ainsi complexe par ses visées et réalisations variées ${ }^{54}$, complexité qui le caractérisera également dans les siècles suivants. Qu'on songe par exemple à ces héros de l'Astrée, de noble extraction mais déguisés en bergers ${ }^{55}$, ou encore aux visées politiques de l'écriture pastorale des XVI ${ }^{\mathrm{e}}$ et XVII ${ }^{\mathrm{e}}$ siècles $^{56}$.

${ }^{53}$ Michel Zink, La Pastourelle, Paris/Montréal, Bordas, 1972, p. 97 : «Dans la pastourelle, la différence de condition entre le chevalier et la bergère frappe d'abord, et l'immoralité de l'aventure. Puis on s'aperçoit que, pour écrire inlassablement le même bizarre poème, il fallait que les poètes fussent toujours à la poursuite d'une même image : celle d'une femme, pur objet de plaisir, suscitée par la nature sauvage et sensuelle, et étrangère à leur sensibilité civilisée ».

${ }^{54}$ Au point que le Centre d'Études de Recherches Comparatistes de Paris VII a pu se demander lors d'une journée d'étude en 2002: "Peut-on parler d'un genre pastoral ?». URL : https:/www.fabula.org/actualites/peut-on-parler-d-un-genrepastoral_4009.php (consulté le 12 février 2019).

${ }^{55}$ Daniel Ménager, L'aventure pastorale, Paris, Les Belles Lettres, 2017, p. 40 et suivantes.

${ }^{56}$ Laurence Giavarini, La distance pastorale: usages politiques de la représentation des bergers (XVI $I^{e} X V I I^{e}$ siècles), Paris, Éditions de l'EHESS, 2010. 
